\title{
A New Approach to Rational Discrete-Time Approximations to Continuous-Time Fractional-Order Systems
}

\author{
Carlos Matos $^{1}$ and Manuel Duarte Ortigueira ${ }^{2}$ \\ ${ }^{1}$ Instituto Politécnico de Setúbal Escola Superior de Tecnologia, Setúbal, Portugal \\ cmatosdest.ips.pt \\ ${ }^{2}$ UNINOVA/DEE Campus da FCT da UNL, Quinta da Torre, 2825-114 \\ Monte da Caparica, Portugal \\ mdo@fct.unl.pt
}

\begin{abstract}
In this paper a new approach to rational discrete-time approximations to continuous fractional-order systems of the form $1 /\left(s^{\alpha}+p\right)$ is proposed. We will show that such fractional-order LTI system can be decomposed into subsystems. One has the classic behavior and the other is similar to a Finite Impulse Response (FIR) system. The conversion from continuous-time to discrete-time systems will be done using the Laplace transform inversion integral and then applying the Bilinear transformation to obtain the corresponding discrete-time system and the corresponding frequency response. An optimization algorithm will be used to find the FIR type parameters that best approximate the discrete-time system.
\end{abstract}

Keywords: Fractional-order, fractional spectrum, discrete time, optimization.

\section{Introduction}

The study and application of fractional systems has been a hot subject for the last years. The heavy mathematical work load that results from the design of continuoustime fractional systems has been a limiting factor to its application [2, 3, 4, 9]. One of the most important research topics in this subject is to obtain simple and effective way to find a discrete-time fractional system that is the equivalent to a defined continuoustime fractional system. The discrete-time systems will inherently simplify this mathematical work load leading to its broader use. One could simply sample the impulse response of a fractional-order continuous-time system, but this approach will inevitably lead to an unstable system [8, 9]. Another possible approach is the conversion from continuous-time to discrete-time by the use of the Bilinear transformation if this leads to stable systems. Inspecting the characteristics of both the impulse response and the spectrum of this converted system it is simple to devise a FIR approximation. This has characteristics that makes it easy to deal with, leads to stable systems and is a good approximation at the same time [11, 12]. The design of the FIR system is done by using an optimization algorithm to find its parameters. 
This paper is organized as follows. In Section 2, the contribution to value creation of this paper is explained. The problem that is addressed is explained in section 3 with a proposed solution and some simulation results, in section 4 some conclusions are drawn and some ideas for future work are explained in section 5.

\section{Contributions to Value Creation}

This paper adds some new insights and ideas on how to decompose a fractional partial fraction system to obtain discrete-time fractional-order systems from the continuoustime counterparts. The use of continuous-time fractional-systems somehow limited due to the mathematical complexity that describes these. This work defines a path that bridges between a continuous-time system and the equivalent discrete-time system. It contributes to a reduction in the necessary mathematical and programmatic work load. This reduced complexity will contribute to a wider use of fractional-systems and may lead to increase the demand for fractional components with the possibility to industrialize the production, opposing to the actual laboratory prototyped reduced production.

\section{Impulse Response of Fractional-Order Systems}

The starting point of our approach will be the differential equation of the type

$$
\sum_{n=0}^{N} a_{n} D^{v_{n}} y(t)=\sum_{m=0}^{M} b_{m} D^{v_{m}} x(t)
$$

where $v$ represents the fractional order of the derivative. Considering that $x(t)=(t)$, we will obtain the impulse response $h(t)$ and one can write:

$$
\sum_{n=0}^{N} a_{n} D^{v_{n}} h(t)=\sum_{m=0}^{M} b_{m} D^{v_{m}} \delta(t)
$$

The general case where the orders are any real is difficult to deal with, we assume that all the $v_{\mathrm{n}}$ are rational or multiples of a $v$. In this commensurate case the transfer function becomes:

$$
H(s)=\frac{\sum_{m=0}^{M} b_{m} s^{m v}}{\sum_{n=0}^{N} a_{n} s^{n v}}=H_{0} \frac{\prod_{m=0}^{M}\left(1-q_{m} z^{-1}\right)}{\prod_{n=0}^{N}\left(1-p_{n} z^{-1}\right)}=H_{0} \prod_{m=0}^{M}\left(1-q_{m} z^{-1}\right)
$$

The third member of equation (3) reflects the particular case of FIR filters considering all $\mathrm{p}_{\mathrm{n}}$ coefficients equal to zero ${ }^{1}$.

\subsection{Simple Fraction with Poles}

As shown above, the transfer function (3) is a sum of partial fractions with the format ${ }^{2}$

$$
F(s)=\frac{1}{\left(s^{v}-p\right)}
$$

\footnotetext{
${ }^{1}$ FIR filters do not have feedback coefficients.

${ }^{2}$ We assume that there are no repeated roots of the denominator.
} 
Replacing the fractional $v$ symbol by the most commonly used $\alpha$ and with $\mathrm{F}$ meaning the Laplace Transform [2], the inversion of a transfer function of type (4) will become

$$
h(t)=\frac{1}{2 \pi j} \int_{a-j \omega}^{a+\omega} F\left(s^{\alpha}\right) e^{s t} d s .
$$

Assuming that

$$
\lim _{s \rightarrow 0} s F(s)=0, \alpha>0
$$

And

$$
\lim _{s \rightarrow \infty} F(s)=0, \alpha>0
$$

In the cut plane $\operatorname{larg}(\mathrm{s}) \mid<\pi$ and also that the function has the poles in the left half complex plane and in the region surrounded by the integration contour the impulse response becomes

$$
h(t)=\sum_{i=1}^{N} R_{i}+\frac{1}{2 \pi j} \int_{-\infty}^{0} F\left(\sigma^{\alpha} e^{-j \alpha \pi}\right) e^{-\sigma} d \sigma+\frac{1}{2 \pi j} \int_{0}^{+\infty} F\left(\sigma^{\alpha} e^{j \alpha \pi}\right) e^{-\sigma} d \sigma
$$

with $R_{i}$ the residues of $F\left(s^{\alpha}\right) e^{\text {st }}$. Using the above expression to invert the partial fraction (4) the solution will be given by

$$
h(t)=\frac{p^{\frac{1}{\alpha-1}}}{\alpha} e^{p^{\frac{1}{\alpha^{t}}}} u(t)+\frac{1}{\pi} \int_{0}^{+\infty} \frac{\sigma^{\alpha} \sin (\alpha \pi)}{\sigma^{2 \alpha}-2 \sigma^{\alpha} p \cos (\alpha \pi)+p^{2}} e^{-\sigma t} d \sigma u(t)
$$

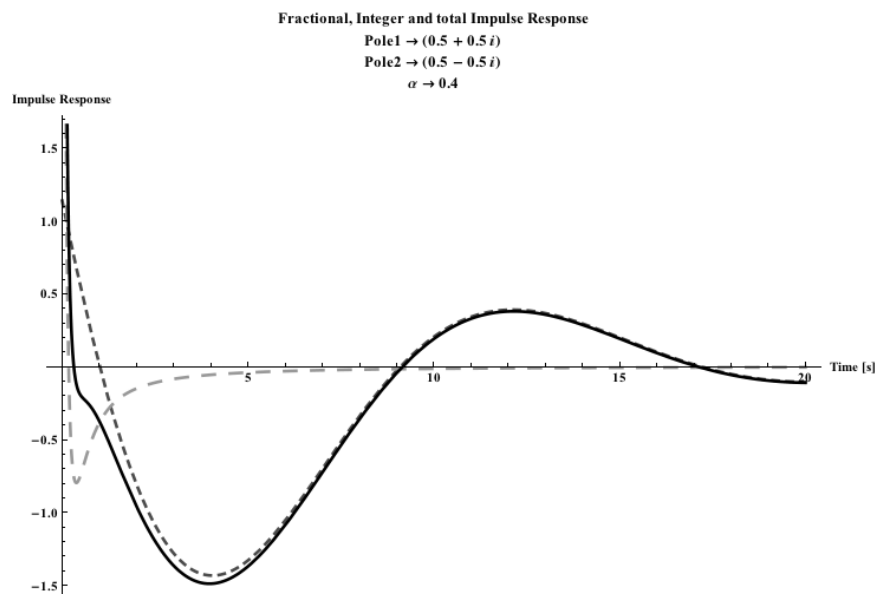

Fig. 1. Fractional part in Light grey large dashed, Integer part in dark grey small dashed and total impulse response in solid black for $\alpha=0.4$ 
Looking into expression (9) one can see that the right hand side has two terms with very different characteristics. The first term is responsible for the short range memory [1] or the integer part of the impulse response, and the second one gives the long range memory or the fractional part of the response. In this paper we will focus only on the case where expression (4) has poles. Working on the principal branch of the multivariate expression $\mathrm{s}^{\alpha}$ within the region $|\arg (p)| \leq \pi \alpha$, the case outside this region $\pi \alpha<|\arg (p)|<\pi$ will not be addressed for now. The representation of this various contributions to the total impulse response is depicted in Fig. 1.

Let's analyze an example of an integrator with a set of complex conjugate poles at locations $\mathrm{p}=0.5 \pm 0.5 \mathrm{j}$. These poles fall in the stability region of a fractional-order system for $\alpha \leq 0.5$ (see Fig. 2). For this value of $\alpha$, the stability frontier will change from the usual $j \omega$ axis to $\pm j \omega \alpha$ lines. From the simple observation of Fig. 1 one can conclude that the impulse response of the fractional system is similar to the response of a FIR filter. With this in mind we will use the Bilinear [7] transformation to convert the fractional integrator to discrete-time and will use a FIR [8] type of expression as an objective function to the optimization algorithm.

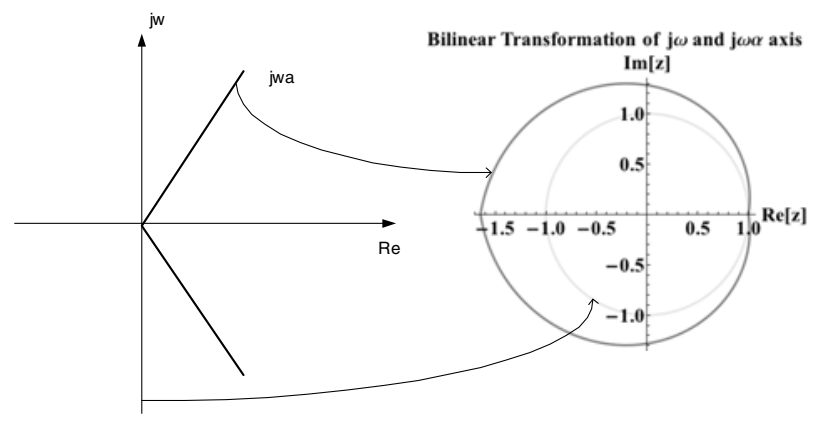

Fig. 2. Bilinear transformation of the $j \omega$ axis in comparison with the Bilinear transformation of $\pm \mathrm{j} \omega \alpha$ the fractional Riemman first sheet region

\subsection{Spectrum Obtained from Bilinear Transformation}

Using the Bilinear transformation, the solution to the inversion integral becomes:

$$
h(t)=\frac{p^{\frac{1}{\alpha-1}}}{\alpha} e^{p^{\frac{1}{\alpha}} t} u(t)+\frac{1}{\pi} \int_{0}^{+\infty} \frac{\sigma^{\alpha} \sin (\alpha \pi)}{\sigma^{2 \alpha}-2 \sigma^{\alpha} p \cos (\alpha \pi)+p^{2}} e^{-\sigma} \cdot \frac{1}{j \frac{2}{T} \tan \left[\omega \frac{T}{2}\right]+\sigma} d \sigma(t)
$$

We will now consider only the fractional part of the spectrum as the integer or short range memory part is already addressed in other publication. This part of the response will be the one used from now on. The Amplitude and phase spectra are depicted in Fig. 3. 


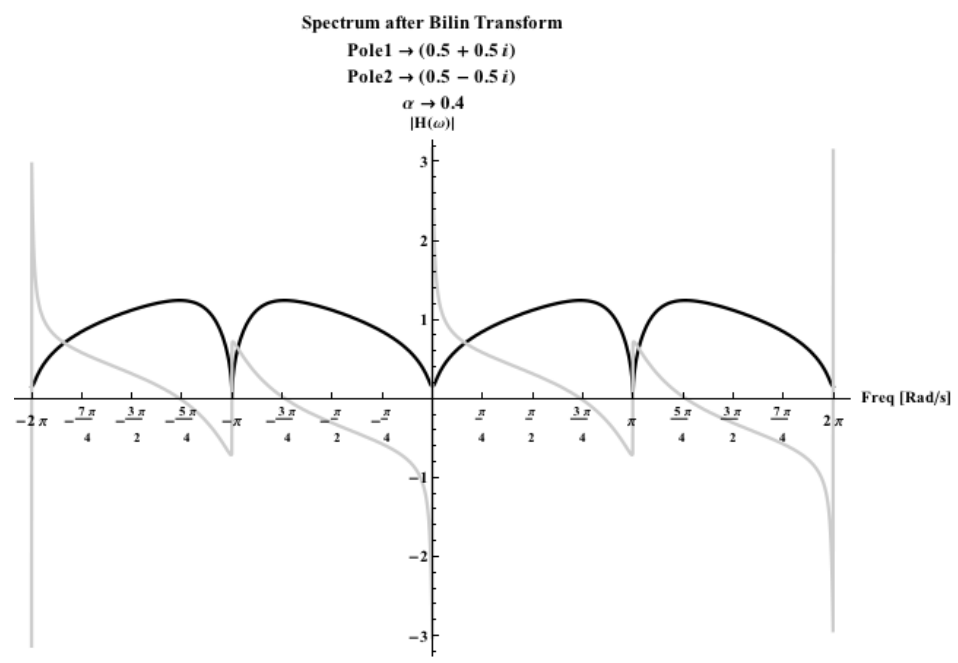

Fig. 3. Spectra of the fractional part resulting from the bilinear transformation. Amplitude is in black and phase in grey.

As we are looking for a FIR with frequency response $\mathrm{H}\left(\mathrm{e}^{\mathrm{j} \omega}\right)$ we will make all $\mathrm{a}_{\mathrm{i}}=0$ in expression (3). With these constrains $\mathrm{H}(\mathrm{z})$ becomes:

$$
H(z)=\sum_{m=0}^{M} b_{m} z^{-1}=H_{0} \prod_{m=0}^{M}\left(1-q_{m} z^{-1}\right)
$$

Now using the Z-Transform to obtain the complex frequency-domain representation of the system we will write

$$
H(z)=\sum_{n=-\infty}^{\infty} x(n) z^{-n}
$$

To achieve our goal, we will use the Fourier transform to rewrite expression (12) in terms $^{3}$ of $\mathrm{z}=\mathrm{e}^{\mathrm{j} \omega \beta}[12]$.

$$
H\left(e^{j \omega \alpha}\right)=\sum_{n=-\infty}^{\infty} q_{n} e^{j \omega \beta}
$$

And to find the FIR filter that better approximates the fractional part of the continuous-time system we will look for the values of the filter coefficients $\mathrm{q}_{\mathrm{i}}$ and the non integer delay $\alpha$. So the idea is to look for a FIR filter with a delay line that has

\footnotetext{
${ }^{3}$ Notice that from now on the fractional parameter of the FIR filter will be referred as $\beta$ to distinguish from the original integer time system $\alpha$.
} 
non integer delays. With these values $[11,12]$ the discrete time response expressed in terms of a summation of Sinc type functions [2, 12].

With this in mind the objective function to minimize will be

$$
\int_{-\pi}^{\pi}\left(\left(\sum_{n=1}^{N_{F I R}} q_{n} e^{j \omega \beta}-H(\omega)\right) \cdot\left(\sum_{n=1}^{N_{F I R}} q_{n} e^{j \omega \beta}-H(\omega)\right)^{*}\right) d \omega
$$

With $\mathrm{H}(\omega)$ the spectra obtained by the bilinear transformation and the symbol $\left(^{*}\right)$ means complex conjugate. Given the good results obtained by the use of the Differential Evolution Genetic Algorithm [10] in optimizing fractional-order filters we will use this same algorithm to find the optimum coefficients. The obtained values will be the $\mathrm{q}_{\mathrm{i}}$ FIR filter coefficients and the value of fractional delay $\beta$.

\subsection{Simulation Results}

For the system described by equation (9) with poles $\mathrm{p}=0.5 \pm 0.5 \mathrm{j}$ and an original chosen $\alpha=0.4$ so to guarantee the system has poles in the first Riemmann sheet the results for the filter coefficients follow and the graphical comparison between the original and obtained amplitudes and phases are depicted in Fig. 4 and Fig. 5.

Total error=6.3903; $\mathrm{q} 1=0.458836 ; \mathrm{q} 2=6.80836, \mathrm{q} 3=-17.2567, \mathrm{q} 4=26.7577, \mathrm{q} 5=-$ 28.9094, $\mathrm{q} 6=21.4203, \mathrm{q} 7=-11.4365, \mathrm{q} 8=4.23464, \mathrm{q} 9=-1.00201, \beta=0.364173$

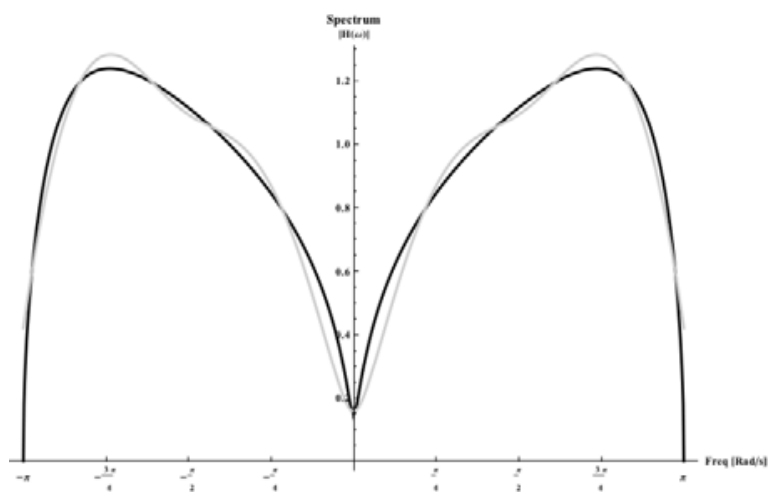

Fig. 4. Comparison of the system spectrum obtained by bilinear transformation and the FIR filter. $H(\omega)$ is in black and FIR in grey

The filter order was chosen by trial and error method. The order of the filter was incremented until the higher order gave a worst result then the immediate lower one. For the present case the best result was obtained with 9 coefficients. 


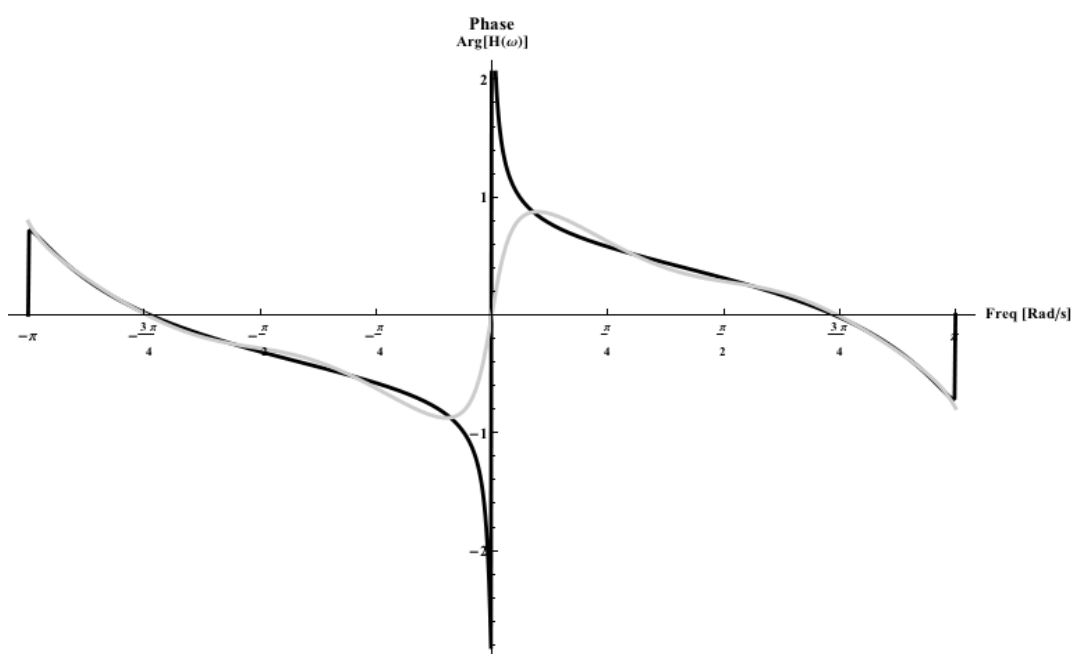

Fig. 5. Comparison of the system phase obtained by bilinear transformation and the FIR filter. $\operatorname{Arg}[\mathrm{H}(\omega)]$ is in black and $\arg [\mathrm{FIR}]$ in grey.

\section{Conclusions}

This work shows a way to obtain a discrete-time system form a known continuoustime. The bilinear (or Tustin) transformation is applicable to convert a continuous time to a discrete-time system using the Laplace inversion integral. We have shown that the FIR type response of the fractional system can be used to obtain a simple filter with a fractional delay/lead that provides a very good approximation. With this approach a path has been drawn to find discrete-time equivalents to the continuoustime fractional-order systems. All the advantages in terms of simplicity and implementation of using a fractional delay/lead discrete-time system become now available.

\section{$5 \quad$ Future Work}

In the present work the order of the FIR filter was determined by trial and error the next step in this subject will be to use the filter order as an optimization parameter. So far the results obtained by applying the method of summation of Sinc type functions to go back to time from the FIR filter is not matured but the results are expected to be published in short time.

\section{References}

1. Ortigueira, M.D.: Fractional Calculus for Scientists and Engineers, 1st edn., vol. 84, p. 152. Springer, Heidelberg (2011) 
2. Ortigueira, M.D.: Introduction to Fractional linear systems. Part2: Discrete-time case. In: Proc.-Vis. Image Signal Processing, vol. 147(1) (February 2000)

3. Barbosa, R.S., Tenreiro Machado, J.A.: Implementation of Discrete-Time Fractional-Order Controllers based on LS Approximations. Acta Polytechnica Hungara, 4 (2006)

4. Ortigueira, M.D.: Introduction to Fractional Linear SystemsI: Continuous-Time case. In: IEEE Proceedings Vision, Image and Signal Processing, vol. 147(1) (2000)

5. Ortigueira, M.D., Serralheiro, A.J.: A new least-squares approach to differintegration modeling. Signal Processing 86, 2582-2591 (2006)

6. Tseng, C.C., Lee, S.L.: Design of adjustable fractional order differentiator using expansion of ideal frequency response. Signal Processing, 498-508 (2012)

7. Ortigueira, M.D., Serralheiro, A.J.: New insights into Pseudo-Fractional ARMA Modeling, Vienna, vol. 1 (September 30, 2004)

8. Koostsookos, P.J., Williamson, R.C.: FIR approximation of Fractional Sample Delay Systems. Analog and Digital Signal Processing. IEEE Transactions on Circuits and Systems-II 43(3) (1996)

9. Tavazoei, M.S.: On Monotonic and Nonmonotonic Step Responses in Fractional Order Systems. IEEE Transactions on Circuits and Systems-II: Express Briefs (2011)

10. Matos, C., Ortigueira, M.D.: Fractional Filters: An Optimization Approach. In: Camarinha-Matos, L.M., Pereira, P., Ribeiro, L. (eds.) DoCEIS 2010. IFIP AICT, vol. 314, pp. 361-366. Springer, Heidelberg (2010)

11. Ortigueira, M.D., Matos, C.: Fractional Discrete-Time Signal Processing: Scale Conversion and Linear Prediction. Nonlinear Dynamics 29, 173-190 (2002)

12. Ortigueira, M.D., Matos, C.: An introduction to the Fractional Linear Prediction. In: Iberoamerican Symposium on Pattern Recognition, pp. 741-748. SIARP, Lisboa (2000) 